Marquette University

e-Publications@Marquette

Exercise Science Faculty Research and

Publications

Exercise Science, Department of

8-2017

\title{
Sex Differences in Spatial Accuracy Relate to the Neural Activation of Antagonistic Muscles in Young Adults
}

\author{
Agostina Casamento-Moran \\ University of Florida - Gainesville \\ Sandra Hunter \\ Marquette University, sandra.hunter@marquette.edu \\ Yen-Ting Chen \\ University of Florida - Gainesville \\ Min-Hyuk Kwon \\ University of Florida - Gainesville \\ Emily J. Fox \\ University of Florida - Gainesville
}

See next page for additional authors

Follow this and additional works at: https://epublications.marquette.edu/exsci_fac

\section{Recommended Citation}

Casamento-Moran, Agostina; Hunter, Sandra; Chen, Yen-Ting; Kwon, Min-Hyuk; Fox, Emily J.; Yacoubi, Basma; and Christou, Evangelos A., "Sex Differences in Spatial Accuracy Relate to the Neural Activation of Antagonistic Muscles in Young Adults" (2017). Exercise Science Faculty Research and Publications. 110. https://epublications.marquette.edu/exsci_fac/110 
Authors

Agostina Casamento-Moran, Sandra Hunter, Yen-Ting Chen, Min-Hyuk Kwon, Emily J. Fox, Basma Yacoubi, and Evangelos A. Christou

This article is available at e-Publications@Marquette: https://epublications.marquette.edu/exsci_fac/110 
Marquette University

e-Publications@Marquette

\section{Exercise Science Faculty Research and Publications/College of Health Sciences}

This paper is NOT THE PUBLISHED VERSION; but the author's final, peer-reviewed manuscript. The published version may be accessed by following the link in th citation below.

Experimental Brain Research, Vol. 235, No. 8 (August 2017): 2425-2436. DOI. This article is (C) SpringerVerlag and permission has been granted for this version to appear in e-Publications@Marquette. Springer-Verlag does not grant permission for this article to be further copied/distributed or hosted elsewhere without the express permission from Springer-Verlag.

\section{Sex differences in spatial accuracy relate to the neural activation of antagonistic muscles in young adults}

Agostina Casamento-Moran

Department of Applied Physiology and Kinesiology, University of Florida, Gainesville, FL Sandra K. Hunter

Department of Physical Therapy, Marquette University, Milwaukee, WI

Yen-Ting Chen

Department of Applied Physiology and Kinesiology, University of Florida, Gainesville, FL Min Hyuk Kwon

Department of Applied Physiology and Kinesiology, University of Florida, Gainesville, FL

Emily J. Fox

Department of Physical Therapy, University of Florida, Gainesville, FL

Brooks Rehabilitation, Jacksonville, FL

Basma Yacoubi

Department of Applied Physiology and Kinesiology, University of Florida, Gainesville, FL 


\section{Evangelos A. Christou}

Department of Applied Physiology and Kinesiology, University of Florida, Gainesville, FL Department of Physical Therapy, University of Florida, Gainesville, FL

\section{Abstract}

Sex is an important physiological variable of behavior, but its effect on motor control remains poorly understood. Some evidence suggests that women exhibit greater variability during constant contractions and poorer accuracy during goal-directed tasks. However, it remains unclear whether motor output variability or altered muscle activation impairs accuracy in women. Here, we examine sex differences in endpoint accuracy during ankle goal-directed movements and the activity of the antagonistic muscles. Ten women ( $23.1 \pm 5.1$ years) and 10 men ( $23 \pm 3.7$ years) aimed to match a target ( $9^{\circ}$ in $180 \mathrm{~ms}$ ) with ankle dorsiflexion. Participants performed 50 trials and we recorded the endpoint accuracy and the electromyographic (EMG) activity of the primary agonist (Tibialis Anterior; TA) and antagonist (Soleus; SOL) muscles. Women exhibited greater spatial inaccuracy (Position error: $t=-2.65, P=0.016$ ) but not temporal inaccuracy relative to men. The motor output variability was similar for the two sexes $(P>0.2)$. The spatial inaccuracy in women was related to greater variability in the coordination of the antagonistic muscles $\left(R^{2} 0.19, P=0.03\right)$. These findings suggest that women are spatially less accurate than men during fast goal-directed movements likely due to an altered activation of the antagonistic muscles.

Keywords

Movement error Motor output variability Electromyography (EMG) Fast movements Men and women

\section{Abbreviations}

CV Coefficient of variation

EMG Electromyography

MVC Maximal voluntary contraction

SOL Soleus

TA Tibialis anterior

\section{Introduction}

In the past two decades, sex has been recognized as an important physiological variable of behavior. Sex differences have been demonstrated for stress response (Noteboom et al. 2001; Christou et al. 2004; Yoon et al. 2009; Keller-Ross et al. 2014), pain perception (Lowery et al. 2003; Feine et al. 1991; Lautenbacher and Strian 1991; Lautenbacher and Rollman 1993; Ellermeier and Westphal 1995; Paulson et al. 1998; Kano et al. 2013; Girard-Tremblay et al. 2014; Labrenz et al. 2015; Mapplebeck et al. 2016; Nahman-Averbuch et al. 2016), and neuromuscular activity during cognitive and motor tasks (Ives et al. 1993; Bell et al. 2006; Lissek et al. 2007; Harwood et al. 2008; Roalf et al. 2014; Pereira et al. 2015; Satterthwaite et al. 2015). However, our understanding of the effect of sex on movement control is at its infancy. Some evidence suggest that women exhibit greater motor output variability during constant force contractions (Yoon et al. 2009; Brown et al. 2010; Vanden Noven et al. 2014; Pereira et al. 2015) and poorer accuracy during complex goal-directed tasks such as throwing and intercepting a ball (Watson and Kimura 1991; Moreno-Briseño et al. 2010). Nonetheless, little is known about whether sex-related differences in accuracy and motor output variability during goal-directed movements exist. Here, we examine whether women exhibit poorer endpoint accuracy and greater motor output variability during single-joint goal-directed movements and the underlying neuromuscular mechanisms. 
Evidence suggests that at normalized levels of force, young women exhibit greater motor output variability relative to young men. This was demonstrated during constant force contractions of the elbow flexors (Yoon et al. 2009; Brown et al. 2010; Pereira et al. 2015) and ankle dorsiflexors (Vanden Noven et al. 2014). Although these findings demonstrate a more variable force output for women while maintaining a constant isometric force, it remains unclear whether women exhibit greater motor output variability and poorer endpoint accuracy during goal-directed movements. Constant force tasks differ from goal-directed tasks in that constant tasks require the steady and continuous production of force while goal-directed tasks require to accurately match a temporal-spatial target. Fast goal-directed movements have been used to understand age-related declines in endpoint control of movement (Chen et al. 2014; Fox et al. 2014; Kwon et al. 2014). For example, movement inaccuracy of older adults has been related to a longer temporal delay of the antagonist relative to the agonist muscle (Fox et al. 2014; Kwon et al. 2014). However, it remains unclear whether sex-related differences exist for movement accuracy and motor output variability during the control of goal-directed movements. Moreover, the underlying neural mechanisms of such differences remain unknown.

The scarce literature on sex differences in the control of goal-directed movements shows that young women exhibit poorer movement accuracy than young men. This is observed when men and women (post puberty) attempt to accurately throw or intercept a ball (Watson and Kimura 1991; Moreno-Briseño et al. 2010). Such sex differences in motor performance could not be explained by sport history, height, or weight (Watson and Kimura 1991). The authors hypothesized that the differences could be due to the women's spatial inaccuracy observed during cognitive tasks (Linn and Petersen 1985; Peters et al. 1995; Voyer et al. 1995; Malinowski 2001; Clint et al. 2012; Estes and Felker 2012; Nazareth et al. 2013; Satterthwaite et al. 2015; Sneider et al. 2015). Therefore, it is not entirely clear whether women exhibit greater spatial inaccuracy compared with men.

In this study, we use an isolated single-joint movement of the ankle to compare movement endpoint accuracy in young men and women. This task is advantageous to more complex tasks, such as throwing a ball, because it allows us to differentiate between spatial and temporal accuracy. In addition, it allows us to determine the neural activation of muscles underlying the sex differences in endpoint accuracy and motor output variability. Thus, the purpose of this study was to determine sex differences in the performance of goal-directed movements in young adults and the underlying neuromuscular mechanisms. We hypothesize that women will exhibit greater spatial inaccuracy than men associated with greater neural variability.

\section{Materials and methods}

\section{Participants}

Ten women ( $23.1 \pm 5.1$ years) and 10 men ( $23 \pm 3.7$ years) participated in the study. All participants reported being healthy without any known neurological impairments and were right-footed (Elias and Bryden 1998). The Institutional Review Board at the University of Florida approved the procedures of this study. All participants signed a written informed consent before participating in the study.

\section{Experimental approach}

Participants completed one experimental session in which they performed an ankle dorsiflexion goal-directed movement task. Each participant performed the following procedures during the session: (1) maximal voluntary contraction (MVC) with ankle dorsiflexor and plantarflexor muscles; (2) 3-5 goal-directed movement practice trials at a different target from the actual target; and (3) 50 goal-directed movement trials with ankle dorsiflexion. Following is a description of the experimental setup and a more detailed description of the experimental procedures. 


\section{Experimental setup}

Participants sat with their left hip joint flexed to $\sim 90^{\circ}$ with $10^{\circ}$ abduction, the knee flexed to $\sim 90^{\circ}$, and the ankle plantarflexed to $\sim 15^{\circ}$. The left foot rested on a customized foot device with an adjustable footplate and was strapped over the metatarsals to ensure a secure position and simultaneous movement between the device and the foot (Fig. 1a). The axis of rotation of the customized foot device was positioned in line with the axis of rotation of the left ankle to allow only dorsiflexion and plantarflexion of the ankle.

a

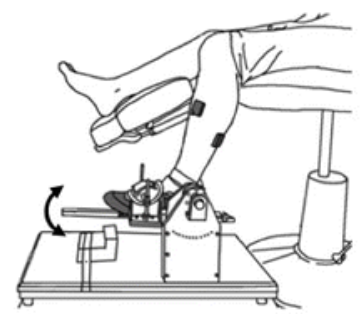

b

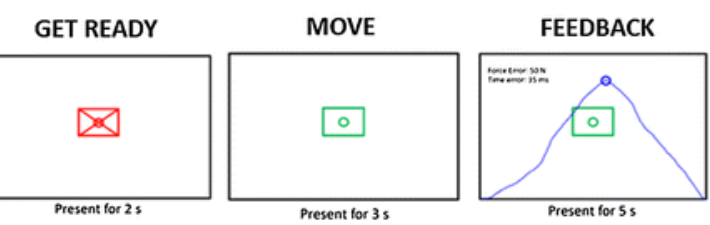

C

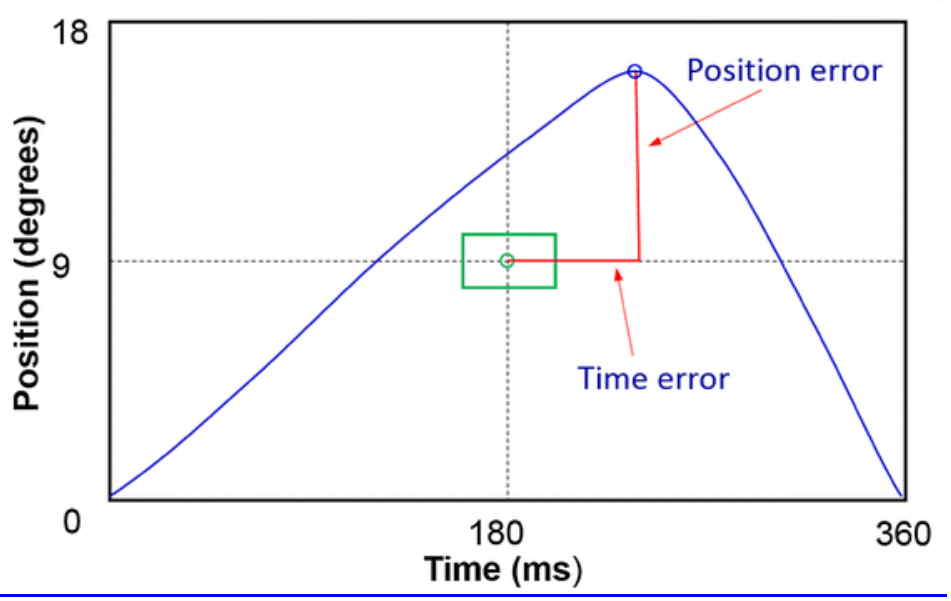

Fig. 1 Experimental setup and goal-directed task. a The left foot was placed and rested on a customized foot device with an adjustable foot plate and secured by a strap over the metatarsals. Participants performed fast upward and downward goal-directed movements with ankle dorsiflexion. $\mathbf{b}$ The task was divided into three phases: GET READY, MOVE, and FEEDBACK. During the GET READY phase, participants viewed a red target on the monitor for $2 \mathrm{~s}$ and remained relaxed. In the MOVE phase participants initiated the movement (no reaction was required) when the target switched from red to green color. The FEEDBACK phase started at the end of the MOVE phase and lasted for $5 \mathrm{~s}$. Participants received visual feedback of their performance (movement trajectory) relative to the target $\left(9^{\circ}-180 \mathrm{~ms}\right)$. c Position error was quantified as the absolute vertical deviation from the target to the peak displacement, whereas time error was quantified as the absolute horizontal deviation from the target to the peak displacement (see "Materials and methods" for more details) (color figure online)

Force The maximum voluntary force exerted during ankle dorsiflexion and plantarflexion was measured with a force transducer (model 41BN, Honeywell, Morristown, NJ, USA). The ankle force signals were high-pass filtered at $0.03 \mathrm{~Hz}$, amplified 50 times (Bridge-8, World Precision Instruments), sampled at $1000 \mathrm{~Hz}$ with a NI-DAQ card (model USB6210, National Instruments), and stored on a personal computer. 
Limb displacement The displacement of the left ankle (dorsiflexion) was measured using a low-friction potentiometer (SP22G-5 K, Mouser Electronics, Mansfield, TX, USA) that was located directly lateral to the fibular malleolus. The ankle position signals were sampled at $1000 \mathrm{~Hz}$ with a NI-DAQ card (Model USB6210, National Instruments, Austin, TX, USA) and stored on a personal computer.

EMG measurements Muscle activation was recorded with a Trigno wireless EMG system (Delsys Inc., Boston, $\mathrm{MA}, \mathrm{USA}$ ) from the tibialis anterior (TA) and soleus (SOL) muscles. The recording electrodes were placed on the skin and in line with the muscle fibers. Specifically, the electrode in the TA was placed at 1/3 on the line between the tip of the fibula and the tip of the medial malleolus. In the SOL the electrode was placed half-way between the end of the head of the gastrocnemius and the origin of the Achilles tendon. The EMG signals were band-pass filtered from 20 to $450 \mathrm{~Hz}$, amplified 1000 times, sampled at $1000 \mathrm{~Hz}$ with a DAQ card (Model USB6210, National Instruments, Austin, TX, USA), and stored on a personal computer.

\section{Experimental procedures}

After the experimental setup, each participant performed the following procedures:

MVC task The MVC was measured for an ankle dorsiflexor muscle (TA; agonist during the goal-directed movements) and a plantarflexor muscle (SOL; antagonists during the goal-directed movements). Each participant was asked to exert and maintain their maximum force for $\sim 3 \mathrm{~s}$. They performed three to five MVCs or until two MVC trials were within 5\% of each other. One-minute rest was provided between consecutive trials. Peak EMG (average of $360 \mathrm{~ms}$ around the peak EMG of the trial) during MVC was recorded and used to normalize the EMG activity during the goal-directed movements.

Goal-directed movements Participants performed unloaded goal-directed movements to accurately match a target by performing a single ankle dorsiflexion. Following 3-5 practice goal-directed movements, each participant performed 50 trials ( 5 blocks of 10 trials each) with $7 \mathrm{~s}$ between trials and 1 min rest between blocks. The goal was to match the peak displacement of the movement (accomplished with an upward and downward movement) with the target. The target consisted of a peak foot displacement of $9^{\circ}$ in $180 \mathrm{~ms}$. We did not provide specific instructions about how to execute the movements allowing participants to choose the motor strategy that worked best for them.

The task involved the following three phases: (1) GET READY, (2) MOVE, and (3) FEEDBACK. The GET READY phase began when a red target was presented on the monitor for $2 \mathrm{~s}$. The red target cued participants to be ready for the MOVE phase. The MOVE phase began when the red target switched to green. The change in target color cued participants to perform the goal-directed movement. The green target stayed on the monitor for $3 \mathrm{~s}$ and participants initiated the movement at their convenience (no reaction time required). The recording of the task began when the participant initiated the movement. The FEEDBACK phase started at the end of the MOVE phase and lasted for $5 \mathrm{~s}$. Participants received visual feedback of their performance (movement trajectory) relative to the target $\left(9^{\circ}-180 \mathrm{~ms}\right)$ (Fig. 1 b). The visual gain was kept constant with a visual angle of $1^{\circ}$ (Vaillancourt et al. 2006).

Data analysis

Data were analyzed off-line using custom-written programs in Matlab ${ }^{\circ}$ (Math Works ${ }^{\mathrm{TM}}$ Inc., Natick, Massachusetts, USA). To compare the neuromuscular control independently from the initial practice-induced adaptations, the last 20 trials were analyzed from each participant (i.e., Block 4 and 5; Fig. 2). 


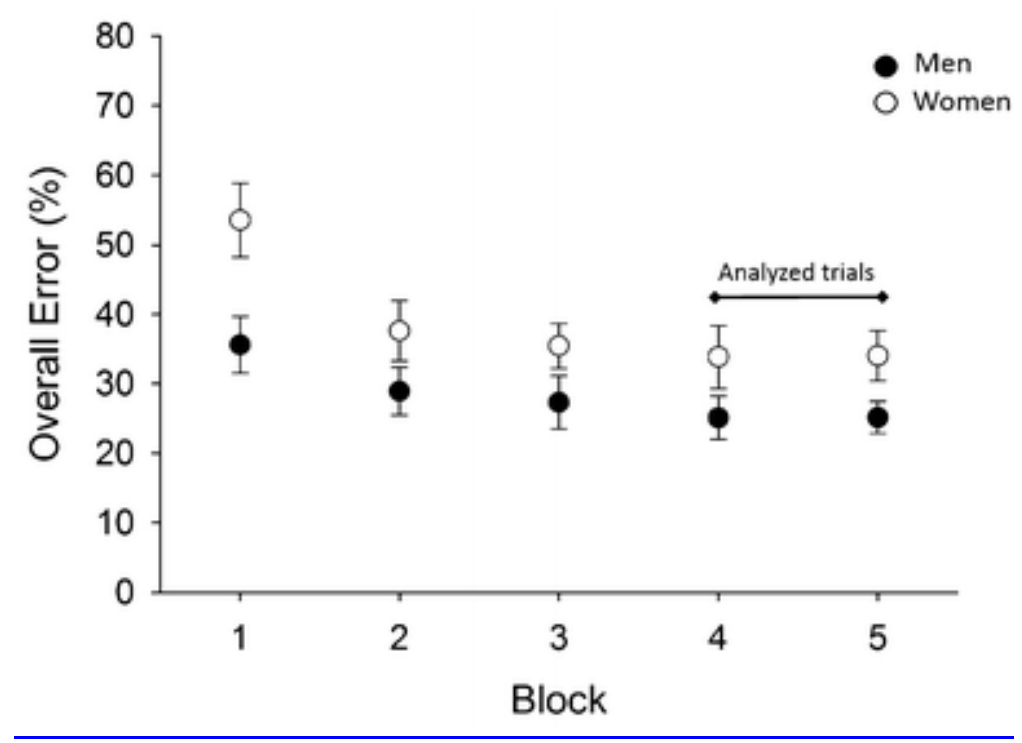

Fig. 2 Analyzed trials. Participants performed a total of 50 practice trials distributed in 5 blocks of 10 trials. Filled and open circles represent the average performance of the 10 trials performed in each block for men and women, respectively. The arrows over the last 2 blocks represent the data used for analysis. The results presented in this manuscript are based on the last 20 practice trials (i.e. 2 blocks) of men and women

Strength We quantified strength with the force produced during the MVC task. From the trials that were within $5 \%$ of each other, we selected the trial of highest force for both dorsiflexion and plantarflexion.

Endpoint error We quantified the position and time error of each trial. Position error was quantified as the absolute vertical deviation from the target to the peak displacement, whereas time error was quantified as the absolute horizontal deviation from the target to the peak displacement (Fig. 1C). We normalized position error to the targeted peak displacement $\left(9^{\circ} ; \mathrm{Eq} . \underline{1}\right)$ and time error to the targeted time-to-peak displacement (180 ms; Eq. 2) to have both variables with the same units (\%).

Positionerror $(\%)=\frac{\text { Peakdisplacementerror }\left({ }^{\circ}\right)}{\text { Targetedpeakdisplacement }\left({ }^{\circ}\right)} \times 100(1)$

Time error $(\%)=\frac{\text { Time to peak displacement error }(\mathrm{ms})}{\text { Targeted time to peak displacement }(\mathrm{ms})} \times 100 .(2)$

Endpoint variability We quantified position and time variability as the coefficient of variation (CV; standard deviation/mean $\times 100$ ) of the position endpoint -absolute horizontal displacement from zero to peak displacement- and time endpoint -absolute vertical displacement from zero to peak displacement.

Trajectory variability To quantify trajectory variability we focus on the variations within the movement from onset to peak. We generated a $1 \mathrm{~Hz}$ low-pass filtered signal (mean trajectory) that we subtracted from a $20 \mathrm{~Hz}$ low-pass filtered signal (raw trajectory). We then normalized the residual trace to the mean trajectory (Trajectory variability; Eq. $\underline{3}$ ).

Trajectory variability $=\frac{(\text { Raw trajectory-mean trajectory) }}{\text { Mean trajectory }} \times 100$. (3)

Neuromuscular activity The interference EMG for each trial was rectified and smoothed with a fourth-order Butterworth digital filter (filtfilt) with a $6 \mathrm{~Hz}$ cutoff frequency (Poston et al. 2008). This filter was used to identify 
the amplitude, onset, and offset of the EMG burst of the primary agonist (TA) and the primary antagonist muscle (SOL) of the ankle.

Muscle activity We examined muscle activity by quantifying the EMG burst in the following ways:

- The EMG burst area was defined as the sum of the EMG values within the EMG burst duration (area under the EMG burst curve) normalized to the sum of EMG recorded during 360 ms around the peak EMG of the MVC task.

- The EMG burst amplitude was defined as the peak EMG activity within the EMG burst duration normalized to the EMG recorded during the MVC.

- The EMG burst duration was defined as the time between EMG onset (15\% of the peak EMG) and EMG offset (15\% of the peak EMG).

- The EMG burst time-to-peak was defined as the time between time of EMG onset (15\% of the peak EMG) and the time of peak EMG activity.

Antagonistic muscle coordination Here, we quantified the average coordination pattern used by men and women in the last 20 trials. Coordination is defined as the amplitude and temporal organization of the two antagonistic muscles. We quantified the coordination in terms of amplitude with TA-SOL peak coactivation (Eq. 4) (Falconer and Winter 1985), whereas we quantified the coordination in terms of time with the TA-SOL temporal coactivation (Eq. $\underline{5}$ ).

TA-SOL peak coactivation(\%) $=\frac{2 \times \text { antagonist } E M G \text { amplitude }}{\text { Agonist }+ \text { antagonist } E M G \text { amplitude }} \times 100$ (4)

\section{TA-SOL temporal coactivation (ms) = TA time of peak EMG (ms) - SOL time of peak EMG(ms). (5)}

See Chen et al. (2014) for a visual representation of the EMG signal processing performed.

Variability of the muscle activity and coordination We determined the variability in the neuromuscular system by quantifying the coefficient of variation (CV) of the EMG measurements of individual muscle activity and coordination across the last 20 trials.

Statistical analysis

The primary dependent variables were: strength: ankle dorsiflexion maximal force $(\mathrm{N})$. Endpoint performance: position endpoint error (\%); Time endpoint error (\%); motor output variability: position endpoint variability (\%); time endpoint variability (\%); trajectory variability (\%). Neural activation of muscle: TA and SOL amplitude (\%); TA and SOL time-to-peak (ms); TA and SOL duration (ms); TA and SOL area (\%); TA and SOL amplitude variability (\%); TA and SOL time-to-peak variability (\%); TA and SOL duration variability (\%); TA and SOL area variability (\%). Antagonistic muscle coordination: TA-SOL peak coactivation (\%); TA-SOL temporal coactivation (ms); TA-SOL peak coactivation variability (\%); TA-SOL temporal coactivation variability (\%).

We used an independent $t$ test to compare men and women for each dependent variable. To examine differences in task acquisition, we used a mixed two-way ANOVA ( 2 sexes $\times 5$ blocks) with repeated measures on blocks. We used bivariate correlations to establish the relationship between strength and position error. Linear regression analyses were used to establish statistical models that could predict position error from the antagonistic muscle activation variables. The goodness-of-fit of each regression was given with the squared correlation ( $R^{2}$; Green and Salkind 2010). All statistical analyses were performed with the IBM statistics 23.0 statistical packages (IBM Inc., New York). We used one-sided $P$ values because we had an a priori hypothesis 
that women will exhibit greater spatial inaccuracy with an altered coordination of the antagonistic muscles. Because of multiple comparisons the alpha level for all statistical tests was adjusted as follows: endpoint performance $-P=0.025$; neural activation of muscle $-P=0.006$. The $P$ value was not adjusted for the antagonistic muscle coordination because the TA-SOL peak coactivation and TA-SOL temporal coactivation were not correlated to each other $\left(R^{2}=0.001 ; P>0.6\right)$. Data are reported as mean $\pm \mathrm{SD}$ within the text and as mean \pm standard error of the mean (SEM) in the figures.

\section{Results}

\section{Strength}

The MVC force prior to the performance of the task for ankle dorsiflexion was 53\% larger for men $(159.79 \pm 39.98 \mathrm{~N})$ compared with women $(103.45 \pm 22.79 \mathrm{~N} ; t=3.87, P<0.01)$. Ankle plantarflexion was similar for men $(127.5 \pm 49 \mathrm{~N})$ and women $(118 \pm 59.03 \mathrm{~N} ; t=0.39, P>0.5)$. Moreover, for both men and women there were no differences in MVC values before and after the task $(P>0.7)$, suggesting that fatigue did not play a role in the results below.

\section{Goal-directed movement control}

Task acquisition Men and women practiced the goal-directed movement 50 times ( 5 blocks $\times 10$ trials each). There was a main effect for $\operatorname{sex}\left(F_{1,18}: 5.49 ; P=0.03\right)$ with men exhibiting greater accuracy than women across the 5 practice blocks. Moreover, there was a main effect for blocks $\left(F_{4,16}: 12.81 ; P<0.01\right)$ with only block 1 being significantly greater in error from all other 4 blocks (all $P<0.02$ ). The rate of task acquisition was similar for men and women (sex $\times$ block interaction; $F_{4,16}: 1.411 ; P=0.24$ ) (Fig. 2). The results that follow are based on the last two blocks ( $2 \times 10$ trials) of practice as they represent the most consistent plateau in performance for both men and women.

Endpoint performance The accuracy of goal-directed movement was quantified with the position error (spatial accuracy) and time error (temporal accuracy). For representative ankle endpoint data of a man and a woman participant see Fig. $\underline{3}$. Women exhibited greater position error $(21.79 \pm 8.85$ vs.

$13.21 \pm 5.19 \% ; t=-2.65, P=0.016$; Fig. 4 a) but similar time error compared with men $(21.50 \pm 10.11$ vs.

$18.96 \pm 6.90 \% ; t=-0.66, P=0.26 ; \mathrm{Fig} . \underline{\mathrm{b}})$. Thus, women exhibit greater spatial inaccuracy than men during fast goal-directed movements.

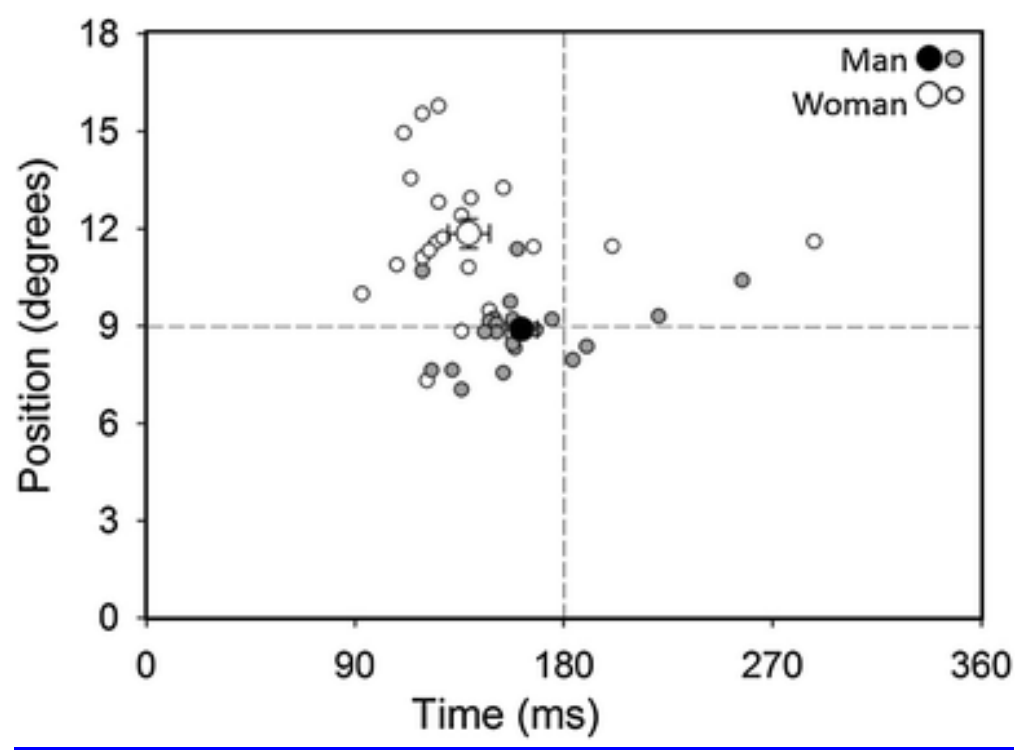


Fig. 3 Overall performance. Representative ankle endpoint data of a man and a woman participant. Small filled (man) and open (woman) circles represent each endpoint of the last 20 trials performed. The large filled (man) and open(woman) circle represent the mean and SD of the movement endpoint of the last 20 trials performed. The interception between the two dashed lines represent the location of the target
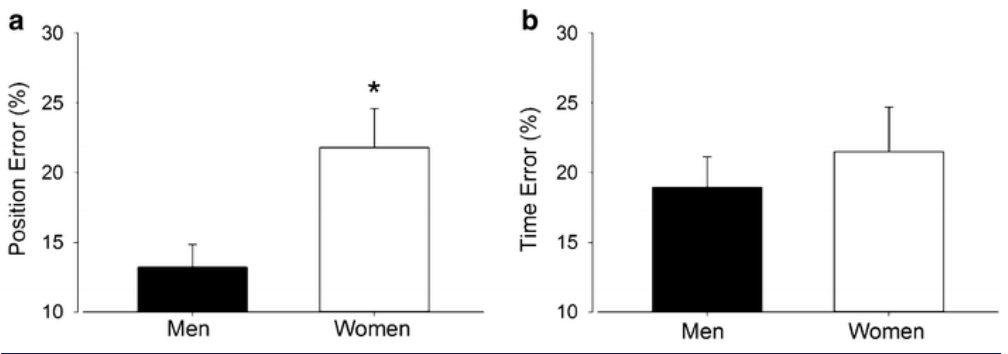

Fig. 4 Spatial and temporal performance. Compared with men, women exhibited greater position error (a) but similar time error (b). Asterisk significant differences between men and women

The greater position error in women relative to men was not associated with participant's strength $\left(R^{2} 0.13, P>0.1\right)$. Thus, the observed accuracy differences between men and women are not due to the observed strength differences.

Motor output variability during goal-directed movements was quantified with the coefficient of variation (CV) of position and time as well as with the trajectory variability. Women and men exhibited similar endpoint variability for both position and time, as well as similar trajectory variability (Table 1 , top). Thus, men and women exhibit similar motor output variability during goal-directed movements.

Table 1 Motor output variability and the neural activation of the antagonistic muscles

\begin{tabular}{|l|l|l|l|}
\hline & Men & Women & P value \\
\hline Motor output variability & Mean \pm SD & Mean \pm SD & \\
\hline Position endpoint variability (\%) & $16.27 \pm 7.1$ & $18.1 \pm 4.9$ & 0.26 \\
\hline Time endpoint variability (\%) & $21.77 \pm 8.2$ & $23.2 \pm 12.0$ & 0.38 \\
\hline Trajectory variability (\%) & $77.2 \pm 7.6$ & $80.3 \pm 16.0$ & 0.59 \\
\hline Neural activation of muscles & & & \\
\hline Tibialis anterior & & & \\
\hline Area (\%) & $18.5 \pm 7.5$ & $20.1 \pm 9.5$ & 0.68 \\
\hline Amplitude (\%) & $43.0 \pm 17.5$ & $48.3 \pm 23.1$ & 0.60 \\
\hline Duration (ms) & $294.7 \pm 25.1$ & $266.1 \pm 47.7$ & 0.11 \\
\hline Time-to-peak (ms) & $155.1 \pm 21.2$ & $139.3 \pm 26.7$ & 0.16 \\
\hline Soleus & & & \\
\hline Area (\%) & $15.3 \pm 10.7$ & $14.0 \pm 12.0$ & 0.80 \\
\hline Amplitude (\%) & $30.0 \pm 22.9$ & $30.3 \pm 33.8$ & 0.98 \\
\hline Duration (ms) & $267.9 \pm 36.1$ & $298.5 \pm 91.1$ & 0.34 \\
\hline Time-to-Peak (ms) & $146.9 \pm 22.5$ & $161.9 \pm 46.1$ & 0.37 \\
\hline Variability in the neural activation of muscles & & & \\
\hline Tibialis anterior & & & \\
\hline Area (\%) & $22.1 \pm 6.5$ & $21.2 \pm 4.0$ & 0.71 \\
\hline Amplitude (\%) & $19.9 \pm 4.7$ & $21.5 \pm 5.5$ & 0.49 \\
\hline Duration (\%) & $22.9 \pm 8.2$ & $23.2 \pm 7.5$ & 0.96 \\
\hline
\end{tabular}




\begin{tabular}{|l|l|l|l|}
\hline Time-to-peak (\%) & $29.6 \pm 8.6$ & $32.9 \pm 12.0$ & 0.49 \\
\hline Soleus & & & \\
\hline Area (\%) & $22.8 \pm 6.3$ & $24.1 \pm 7.4$ & 0.08 \\
\hline Amplitude (\%) & $31.2 \pm 12.2$ & $39.5 \pm 12.4$ & 0.68 \\
\hline Duration (\%) & $29.08 \pm 10.17$ & $38.41 \pm 12.41$ & 0.15 \\
\hline Time-to-peak (\%) & $41.27 \pm 12.83$ & $53.33 \pm 17.53$ & 0.09 \\
\hline
\end{tabular}

Means and standard deviations of all the variables quantifying motor output variability (top), neural activation of muscles (middle) and variability in the neural activation of muscles (bottom)

\section{Neural activation of muscles}

We quantified the neural activation of the antagonistic muscles with the mean activation and the variability of the area, amplitude, duration, and time-to-peak of the EMG burst for each muscle. The area, amplitude, duration, and time-to-peak of the TA and SOL muscles (Table 1, middle), as well as the variability in the same measurements (Table $\underline{1}$, bottom), was similar for men and women. Thus, men and women exhibited similar activation of the antagonistic muscles.

\section{Antagonistic muscle coordination}

We quantified the coordination between the antagonistic muscles (TA and SOL) with the mean and variability of the TA-SOL peak coactivation and TA-SOL temporal coactivation. For the mean antagonistic muscle coordination women exhibited greater EMG peak temporal coactivation than men $(67.10 \pm 82.04$ vs.

$19.71 \pm 22.71 \mathrm{~ms} ; t=-1.76, P=0.04$ ) (Fig. $\underline{5}$, top panel). For the variability of the antagonistic muscle coordination, women exhibited greater TA-SOL peak coactivation (19.02 \pm 10.05 vs.

$12.72 \pm 2.93 \% ; t=-1.90, P=0.03$ ) than men (Fig. $\underline{5}$, bottom panel). Thus, women exhibited altered temporal coordination between the antagonistic muscles and greater variability in the coordination of the amplitude of the antagonistic muscles.

\section{Antagonistic Muscle Coordination}

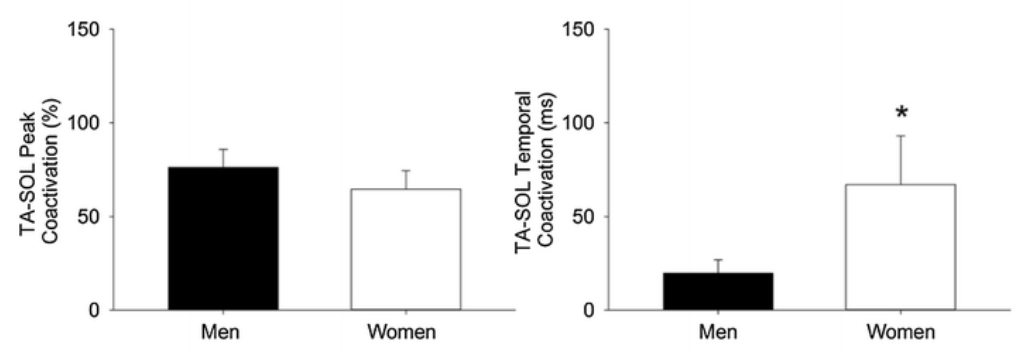

Variability of Antagonistic Muscle Coordination

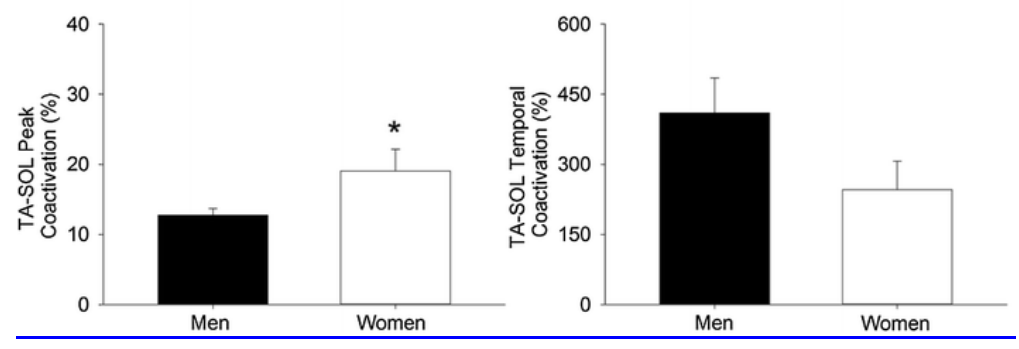

Fig. 5 Antagonistic muscle coordination. Women exhibited greater TA-SOL temporal coactivation (top right panel) and greater the TA-SOL peak coactivation variability (bottom left panel) compared with men. Asterisk significant differences between men and women 
Correlation between neural activation of muscles and position errorWomen were less spatially accurate than men during goal-directed movements with the ankle. We performed a linear regression analysis to predict the position error in men and women from all the coordination EMG variables independently. Only the TA-SOL peak coactivation variability predicted position error $\left(R^{2} 0.19, P=0.03\right.$; Fig. $\left.\underline{6}\right)$. Thus, greater variability in the coordination of the amplitude of the antagonistic muscles is associated with sex-related differences in spatial inaccuracy.

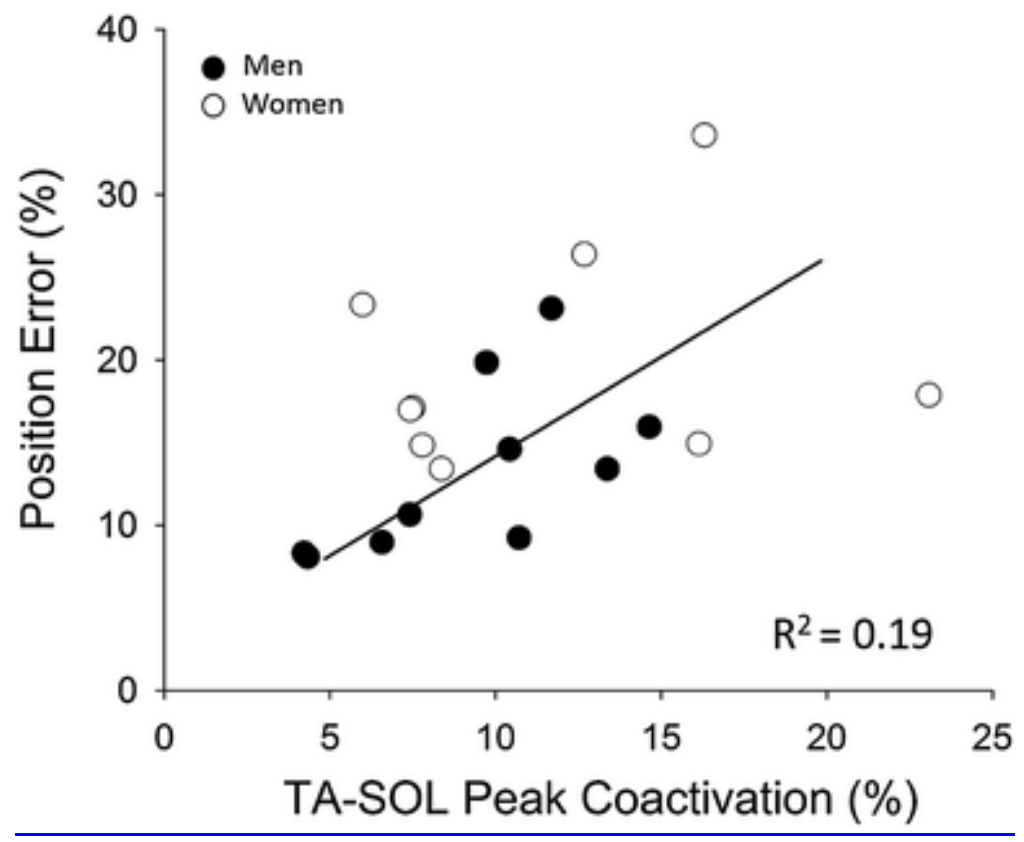

Fig. 6 Variability of the antagonistic muscle coordination. EMG peak coactivation variability predicted position error in men and women $\left(R^{2}=0.19, P=0.03\right)$. Participants who exhibited greater variability in their antagonistic peak coactivation (primarily women) exhibited a greater position error

\section{Discussion}

The purpose of this study was to examine sex-related differences in the performance of goal-directed movements in young adults. Women exhibited greater spatial inaccuracy (Position error), but similar motor output variability compared with men. The greater spatial inaccuracy in women was related to an altered activation of the antagonistic muscles. Specifically, women demonstrated lesser temporal coactivation than men and greater amplitude coactivation variability of the antagonistic muscles. These results provide supporting evidence to the hypothesis (Watson and Kimura 1991) that, during goal-directed movements, women exhibit greater spatial inaccuracy than men and demonstrate that the spatial inaccuracy in women is related to an altered antagonistic muscle coordination.

\section{Sex differences in motor control}

Only in the last 15-20 years, scientists and clinicians have begun to understand sex as an important physiological variable associated with behavior and disease (Beery and Zucker 2011; Miller, 2012, 2014; Fuller and Insel 2014). Because many of the studies - in both animal and human research-include only men, the sex bias in biomedical and movement science remains large (Beery and Zucker 2011; Miller 2012; Hunter 2016). Given the paucity of data on sex-related differences in the control of goal-directed movements, this study presents our initial efforts to determine whether such differences exist. 
To date, ambiguity exists on whether or not sex-related differences in cognitive (Duff and Hampson 2001; Postma et al. 2004; Lejbak et al. 2009) and motor performance (Lomond and Côté 2010; Endo and Kawahara 2011; Nedelec et al. 2011; Emery and Côté 2012; Fedorowich et al. 2013; Messing et al. 2014; Vafadar et al. 2015; Almuklass et al. 2016) exists. However, our results support and extend previous studies, which demonstrate a better spatial accuracy for men in certain cognitive (Linn and Petersen 1985; Peters et al. 1995; Voyer et al. 1995; Malinowski 2001; Clint et al. 2012; Estes and Felker 2012; Nazareth et al. 2013) and motor (Watson and Kimura 1991) tasks. Specifically, when men and women were asked to determine spatial relations despite distracting information or to rotate quickly and accurately 2-and 3-dimensional figures in imagination, men outperformed women in spatial perception and mental rotation (Linn and Petersen 1985; Voyer et al. 1995). Furthermore, men exhibit better performance during complex goal-directed movement tasks, such as throwing and intercepting a ball (Watson and Kimura 1991; Moreno-Briseño et al. 2010). Based on the cognitive (Linn and Petersen 1985; Peters et al. 1995; Voyer et al. 1995; Malinowski, 2001; Clint et al. 2012; Nazareth et al. 2013) findings and the absence of any sport history differences or physical characteristics, the sex differences in movement performance were hypothesized to be due to spatial inaccuracy in women (Watson and Kimura 1991). Nonetheless, this interpretation was limited by the complexity of the movement tasks. Throwing and intercepting a ball involves the coordination of many degrees of freedom (e.g., muscles and joints) and multiple systems (e.g., visual and motor). Our study, therefore, is the first to directly address such hypothesis. Our goal-directed task allows us to distinguish between spatial (position error) and temporal (time error) control of the performed movement. We found that men are spatially more accurate than women (position error; Fig. 4a) and that temporal accuracy (time error; Fig. 4b) is similar between the groups. Our findings, therefore, provide evidence to the hypothesis (Watson and Kimura 1991) that, during goal-directed movements, young men are more accurate than women due to better spatial accuracy.

Contrary to our expectations, we found no differences in motor output variability. Specifically, the coefficient of variation (CV) of position and CV of time as well as the trajectory variability were similar for men and women. Our expectations were based on previous findings, where men and women performed constant force contractions with the elbow flexors (Yoon et al. 2009; Brown et al. 2010; Pereira et al. 2015) and ankle dorsiflexors (Vanden Noven et al. 2014). These findings could be different from ours for the following reasons: (1) constant contractions are different from the goal-directed movements that we used in the current study. Constant contractions required men and women to track a line, whereas our goal-directed movements required to accurately reach a temporal-spatial target (endpoint accuracy) across several trials. (2) Constant contractions require continuous visuomotor control accomplished through online corrections. In our goal-directed task, the temporal target $(180 \mathrm{~ms})$ doesn't allow for online corrections and the motor output represent the execution of the preplanned motor command. Therefore, these methodological differences could explain the incongruent findings between our study and previous studies (Yoon et al. $\underline{2009}$; Brown et al. 2010; Vanden Noven et al. 2014; Pereira et al. 2015).

\section{Sex differences in the coordination of antagonistic muscles}

Our results provide novel evidence that an altered coordination of the antagonistic muscles impairs spatial accuracy in women. We found that compared with men, women exhibited lesser temporal coactivation (Fig. $\underline{5}$, top panel) and more variable amplitude coactivation (Fig. $\underline{5}$, bottom panel) of the antagonistic muscles. Moreover, we were able to predict the greater spatial inaccuracy in women relative to men from greater variability in the coordination of the antagonistic muscles. Specifically, we found that greater variability in the amplitude coactivation of the antagonistic muscles predicted position error in men and women (Fig. $\underline{6}$ ).

Our results trigger the following question: How is the impaired spatial accuracy in women related to an altered activation of the antagonistic muscles? There is evidence that when the task requires more spatial precision, participants increase the coactivation of the antagonistic muscles (Gribble et al. 2003). This is supported by 
aging studies during similar goal-directed movements to ours. Specifically, the impaired endpoint accuracy of older adults relative to young adults is predicted by lesser coactivation of antagonistic muscles (Kwon et al. 2014). Here, we demonstrate that women exhibit lesser temporal coactivation and more variable amplitude coactivation of the antagonistic muscles than men. Thus, this altered coordination of the antagonistic muscles could lead to greater spatial errors in women by changing the optimal coactivation scheme required for this type of movement.

The altered activation of antagonistic muscles in women could be related to the well-established sexual dimorphism of the central nervous system observed through development (MacLusky and Naftolin 1981). Although the exact mechanism remains unknown, it is possible that the sex-related differences in brain maturation (De Bellis et al. 2001; Koolschijn and Crone 2013) and connectivity (Sun et al. 2015) as well as the differential activation of cortical areas during motor tasks (Lissek et al. 2007) underlie the altered neuromuscular activation in women. Future studies should explore more in depth the differential neuromuscular activation in women as well as its consequences in motor control.

Finally, we found similar net activation of individual muscles between women and men. Specifically, the amplitude, duration, and time-to-peak of both the agonist (TA) and antagonist (SOL) muscles was similar between men and women. This contrasts previous results suggesting sex-related differences in the neural activation of muscle during rapid elbow flexion movements (Ives et al. 1993). The following present several experimental differences that could explain the incongruent results: (1) the limb used for the two studies is different. The previous study used the upper limb, whereas we used the lower limb. (2) We targeted a submaximal movement time of $180 \mathrm{~ms}$, whereas Ives et al. (1993) targeted the fastest possible movement time; (3) the movements performed in our experiment and that of Ives et al. (1993) were loaded differently; (4) we analyzed the last 20 trials of a 50 practice trial session, while they had participants perform 10 trials for each condition and analyzed all the performed trials (Ives et al. 1993). Regardless of the exact differences between the two studies, we believe that the endpoint of goal-directed movements is the result of the coordination of the involved antagonistic muscles and not simply caused by the altered activation of a single muscle. Support for this conclusion comes from numerous studies that examine the triphasic activation of antagonistic muscles and effect on movement behavior (Gottlieb et al. 1992, 1996; Almeida et al. 1995; Christou et al. 2007).

\section{Limitations}

Our findings are based on discrete ankle dorsiflexion movements and it is not clear whether our findings will apply to multi-joint movements. However, the performance of similar fast goal-directed contractions predicted global functional capacity of individuals with Spinocerebellar Ataxia Type 6 (SCA6) (Casamento-Moran et al. 2015), suggesting that this task is a good model for understanding the control of more complex movements. Moreover, in this study we did not account for women's menstrual cycle. These findings likely represent the average sex differences in motor control independent of the phase in the menstrual cycle. Sex-related differences in the control of movement could be different depending on the specific phase of the menstrual cycle (Peters et al. 1995; Hunter 2016). Finally, our study addressed sex differences in young adults during ankle goal-directed movements towards one single target. Future studies should address whether these sex differences hold in other populations, such as pre-pubertal children and older adults, and in goal-directed movements with different limbs and targets.

\section{Conclusion}

The literature of sex-related differences and motor control is in its infancy. This study represents our first attempt to examine sex differences in motor control using a well-controlled goal-directed movement task. Our findings provide novel evidence that men are spatially more accurate than women during a goal-directed movement task due to an altered coordination of the antagonistic muscles. Although these results further our 
understanding of the sex differences in motor control, more studies are needed to better understand the healthy and diseased motor system in men and women.

\section{References}

Almeida GL, Hong DA, Corcos D, Gottlieb GL (1995) Organizing principles for voluntary movement: extending single-joint rules. J Neurophysiol 74:1374-1381

Almuklass AM, Price RC, Gould JR, Enoka RM (2016) Force steadiness as a predictor of time to complete a pegboard test of dexterity in young men and women. J Appl Physiol 120:1410-1417

Beery AK, Zucker I (2011) Sex bias in neuroscience and biomedical research. Neurosci Biobehav Rev 35:565-572

Bell EC, Willson MC, Wilman AH, Dave S, Silverstone PH (2006) Males and females differ in brain activation during cognitive tasks. Neuroimage 30:529-538

Brown RE, Edwards DL, Jakobi JM (2010) Sex differences in force steadiness in three positions of the forearm. Eur J Appl Physiol 110:1251-1257

Casamento-Moran A, Chen Y-T, Kwon M, Snyder A, Subramony SH, Vaillancourt DE, Christou EA (2015) Force dysmetria in spinocerebellar ataxia 6 correlates with functional capacity. Front Hum Neurosci 9:1-7

Chen Y-T, Kwon M, Fox EJ, Christou EA (2014) Altered activation of the antagonist muscle during practice compromises motor learning in older adults. J Neurophysiol 112:1010-1019

Christou EA, Jakobi JM, Critchlow A, Fleshner M, Enoka RM (2004) The 1- to 2-Hz oscillations in muscle force are exacerbated by stress, especially in older adults. J Appl Physiol 97:225-235

Christou EA, Poston B, Enoka JA, Enoka RM (2007) Different neural adjustments improve endpoint accuracy with practice in young and old adults. J Neurophysiol 97:3340-3350

Clint EK, Sober E, Garland T, Rhodes JS (2012) Male superiority in spatial navigation: adaptation or side effect? Q Rev Biol 87:289-313

De Bellis MD, Keshavan MS, Beers SR, Hall J, Frustaci K, Masalehdan A, Noll J, Boring AM (2001) Sex differences in brain maturation during childhood and adolescence. Cereb Cortex 11:552-557

Duff SJ, Hampson E (2001) A sex difference on a novel spatial working memory task in humans. Brain Cogn 47:470-493

Elias L, Bryden MP (1998) Footedness is a better predictor of language lateralisation than handedness. Laterality 3:41-51

Ellermeier W, Westphal W (1995) Gender differences in pain ratings and pupil reactions to painful pressure stimuli. Pain 61:435-439

Emery K, Côté JN (2012) Repetitive arm motion-induced fatigue affects shoulder but not endpoint position sense. Exp Brain Res 216:553-564

Endo H, Kawahara K (2011) Gender differences in hand stability of normal young people assessed at low force levels. Ergonomics 54:273-281

Estes Z, Felker S (2012) Confidence mediates the sex difference in mental rotation performance. Arch Sex Behav 41:557-570

Falconer K, Winter DA (1985) Quantitative assessment of co-contraction at the ankle joint in walking. Electromyogr Clin Neurophysiol 25:135-149

Fedorowich L, Emery K, Gervasi B, Côté JN (2013) Gender differences in neck/shoulder muscular patterns in response to repetitive motion induced fatigue. J Electromyogr Kinesiol 23:1183-1189

Feine JS, Bushnell MC, Miron D, Duncan GH (1991) Sex differences in the perception of noxious heat stimuli. Pain 44:255-262

Fox EJ, Moon H, Kwon M, Chen Y-T, Christou EA (2014) Neuromuscular control of goal-directed ankle movements differs for healthy children and adults. Eur J Appl Physiol 114:1889-1899

Fuller CM, Insel PA (2014) I don't know the question, but sex is definitely the answer! Focus on "In pursuit of scientific excellence: sex matters" and "Do you know the sex of your cells?". Am J Physiol Cell Physiol 306:C1-C2

Girard-Tremblay L, Auclair V, Daigle K, Léonard G, Whittingstall K, Goffaux P (2014) Sex differences in the neural representation of pain unpleasantness. J Pain 15:867-877 
Gottlieb GL, Latash ML, Corcos DM, Liubinskas TJ, Agarwal GC (1992) Organizing principles for single joint movements: V. Agonist-antagonist interactions. J Neurophysiol 67:1417-1427

Gottlieb GL, Song Q, Hong DA, Corcos DM (1996) Coordinating two degrees of freedom during human arm movement: load and speed invariance of relative joint torques. J Neurophysiol 76:3196-3206

Green SB, Salkind NJ (2010) Using SPSS for windows and macintosh: analyzing and understanding data. Prentice Hall Press

Gribble PL, Mullin LI, Cothros N, Mattar A (2003) Role of cocontraction in arm movement accuracy. J Neurophysiol 89:2396-2405

Harwood B, Edwards DL, Jakobi JM (2008) Age- and sex-related differences in muscle activation for a discrete functional task. Eur J Appl Physiol 103:677-686

Hunter SK (2016) The relevance of sex differences in performance fatigability. Med Sci Sports Exerc 48:22472256

Ives JC, Kroll WP, Bultman LL (1993) Rapid movement kinematic and electromyographic control characteristics in males and females. Res Q Exerc Sport 64:274-283

Kano M, Farmer AD, Aziz Q, Giampietro VP, Brammer MJ, Williams SCR, Fukudo S, Coen SJ (2013) Sex differences in brain response to anticipated and experienced visceral pain in healthy subjects. Am J Physiol Gastrointest Liver Physiol 304:G687-G699

Keller-Ross ML, Pereira HM, Pruse J, Yoon T, Schlinder-Delap B, Nielson KA, Hunter SK (2014) Stressor-induced increase in muscle fatigability of young men and women is predicted by strength but not voluntary activation. J Appl Physiol 116:767-778

Koolschijn PCMP, Crone EA (2013) Sex differences and structural brain maturation from childhood to early adulthood. Dev Cogn Neurosci 5:106-118

Kwon M, Chen Y-T, Fox EJ, Christou EA (2014) Aging and limb alter the neuromuscular control of goal-directed movements. Exp Brain Res 232:1759-1771

Labrenz F, Icenhour A, Thürling M, Schlamann M, Forsting M, Timmann D, Elsenbruch S (2015) Sex differences in cerebellar mechanisms involved in pain-related safety learning. Neurobiol Learn Mem 123:92-99

Lautenbacher S, Rollman GB (1993) Sex differences in responsiveness to painful and non-painful stimuli are dependent upon the stimulation method. Pain 53:255-264

Lautenbacher S, Strian F (1991) Sex differences in pain and thermal sensitivity: the role of body size. Percept Psychophys 50:179-183

Lejbak L, Vrbancic M, Crossley M (2009) The female advantage in object location memory is robust to verbalizability and mode of presentation of test stimuli. Brain Cogn 69:148-153

Linn MC, Petersen AC (1985) Emergence and characterization of sex differences in spatial ability: a metaanalysis. Child Dev 56:1479-1498

Lissek S, Hausmann M, Knossalla F, Peters S, Nicolas V, Güntürkün O, Tegenthoff M (2007) Sex differences in cortical and subcortical recruitment during simple and complex motor control: an fMRI study. Neuroimage 37:912-926

Lomond KV, Côté JN (2010) Movement timing and reach to reach variability during a repetitive reaching task in persons with chronic neck/shoulder pain and healthy subjects. Exp Brain Res 206:271-282

Lowery D, Fillingim RB, Wright RA (2003) Sex differences and incentive effects on perceptual and cardiovascular responses to cold pressor pain. Psychosom Med 65:284-291

MacLusky N, Naftolin F (1981) Sexual differentiation of the central nervous system. Science (80-.) 211:12941302

Malinowski JC (2001) Mental rotation and real-world wayfinding. Percept Mot Skills 92:19-30

Mapplebeck JCS, Beggs S, Salter MW (2016) Sex differences in pain: a tale of two immune cells. Pain 157(Suppl):S2-S6

Messing K, Stock S, Côté J, Tissot F (2014) Is sitting worse than static standing? How a gender analysis can move us toward understanding determinants and effects of occupational standing and walking. J Occup Environ Hyg 9624:D11-D17 
Miller VM (2012) In pursuit of scientific excellence: sex matters. Am J Physiol Endocrinol Metab 302:E1025E1026

Miller VM (2014) Why are sex and gender important to basic physiology and translational and individualized medicine? Am J Physiol Heart Circ Physiol 306:H781-H788

Moreno-Briseño P, Díaz R, Campos-Romo A, Fernandez-Ruiz J (2010) Sex-related differences in motor learning and performance. Behav Brain Funct 6:74

Nahman-Averbuch H, Dayan L, Sprecher E, Hochberg U, Brill S, Yarnitsky D, Jacob G (2016) Sex differences in the relationships between parasympathetic activity and pain modulation. Physiol Behav 154:40-48

Nazareth A, Herrera A, Pruden SM (2013) Explaining sex differences in mental rotation: role of spatial activity experience. Cogn Process 14:201-204

Nedelec B, Dion K, Correa JA, Desrosiers J (2011) Upper extremity performance test for the elderly (TEMPA): normative data for young adults. J Hand Ther 24:31-43

Noteboom JT, Fleshner M, Enoka RM (2001) Activation of the arousal response can impair performance on a simple motor task. J Appl Physiol 91:821-831

Paulson PE, Minoshima S, Morrow TJ, Casey KL (1998) Gender differences in pain perception and patterns of cerebral activation during noxious heat stimulation in humans. Pain 76:223-229

Pereira HM, Spears VC, Schlinder-Delap B, Yoon T, Nielson KA, Hunter SK (2015) Age and sex differences in steadiness of elbow flexor muscles with imposed cognitive demand. Eur J Appl Physiol 115:1367-1379

Peters M, Laeng B, Latham K, Jackson M, Zaiyouna R, Richardson C (1995) A redrawn Vandenberg and Kuse mental rotations test: different versions and factors that affect performance. Brain Cogn 28:39-58

Postma A, Jager G, Kessels RPC, Koppeschaar HPF, Van Honk J (2004) Sex differences for selective forms of spatial memory. Brain Cogn 54:24-34

Poston B, Enoka JA, Enoka RM (2008) Practice and endpoint accuracy with the left and right hands of old adults: the right-hemisphere aging model. Muscle Nerve 37:376-386

Roalf DR, Gur RE, Ruparel K, Calkins ME, Satterthwaite TD, Bilker WB, Hakonarson H, Harris L, Gur RC (2014) Within-individual variability in neurocognitive performance: age- and sex-related differences in children and youths from ages 8 to 21 . Neuropsychology 28:506-518

Satterthwaite TD, Wolf DH, Roalf DR, Ruparel K, Erus G, Vandekar S, Gennatas ED, Elliott MA, Smith A, Hakonarson H, Verma R, Davatzikos C, Gur RE, Gur RC (2015) Linked sex differences in cognition and functional connectivity in youth. Cereb Cortex 25:2383-2394

Sneider JT, Hamilton DA, Cohen-Gilbert JE, Crowley DJ, Rosso IM, Silveri MM (2015) Sex differences in spatial navigation and perception in human adolescents and emerging adults. Behav Process 111:42-50

Sun Y, Lee R, Chen Y, Collinson S, Thakor N, Bezerianos A, Sim K (2015) Progressive gender differences of structural brain networks in healthy adults: a longitudinal, diffusion tensor imaging study. PLoS ONE 10:e0118857

Vafadar AK, Côté JN, Archambault PS (2015) Sex differences in the shoulder joint position sense acuity: a crosssectional study. BMC Musculoskelet Disord 16:273

Vaillancourt DE, Haibach PS, Newell KM (2006) Visual angle is the critical variable mediating gain-related effects in manual control. Exp Brain Res 173:742-750

Vanden Noven ML, Pereira HM, Yoon T, Stevens AA, Nielson KA, Hunter SK (2014) Motor variability during sustained contractions increases with cognitive demand in older adults. Front Aging Neurosci 6:97

Voyer D, Voyer S, Bryden MP (1995) Magnitude of sex differences in spatial abilities: a meta-analysis and consideration of critical variables. Psychol Bull 117:250-270

Watson NV, Kimura D (1991) Nontrivial sex differences in throwing and intercepting: relation to psychometrically-defined spatial functions. Pers Individ Dif 12:375-385

Yoon T, Keller ML, De-Lap BS, Harkins A, Lepers R, Hunter SK (2009) Sex differences in response to cognitive stress during a fatiguing contraction. J Appl Physiol 107:1486-1496 